\title{
Automation the Process of Unifying the Change in the Firewall Performance
}

\author{
Kirandeep Kaur \\ Department of Computer \\ Science and Engineering \\ Lovely Professional University, \\ Phagwara
}

\begin{abstract}
The rapid growth of internet leads to increase in the number of attacks resulting in malicious data to enter in the system. Firewall is introduced so as to resist from the attacks. Anomalies are being generated as rules that are defined may result in conflicts. For that reason an effective anomaly detection and resolution approach is needed and after resolving conflicts, the rules can be reordered dynamically that improve the efficiency of anomaly management framework. Firewall log analysis has been done and then from that analysis primitive rules are defined. They planned the safety policy found on the rules described by the network administrator that decided which packet can be passed to an organizations private network. In addition, analyze the content of the logged data to detect the irrelevant behavior. The logs showing irrelevant behavior are blocked with the access so as to add more security to the network.
\end{abstract}

\section{Keywords}

Policy anomaly, Firewall, Firewall log analysis, Internet, Attacks

\section{INTRODUCTION}

Computer security is as useful to the devices like as computer networks such as internal or external together with the internet. This field includes all the procedure and techniques, by which PC based equipment, information and a services, are protected from unintentional or illegal access, demolition and is of increasing significance in line with the increasing confidence on computer systems of most society. Firewall main purpose is to secure the network from unlawful users that can harm the services provided by the private networks linked by the Internet [1]. All messages that is incoming or leaving the intranet surpass throughout the firewall and it's will examine each and every message and block them that do not meet the specific protection criterion. Firewall policy has set of rules that are defined by the administrator which has some<condition, action>. A huge amount of policy regulations matches the same packet in firewall policy then it leads to firewall policy anomalies (conflicts).Firewall policies comprise a repetition of policy rules which are performed on the packets and that perform the desired actions [6]. The design for the identification is based on the specific condition and the rules.

The phrase condition situation in a rule depicts a group of field as to recognize a certain type of packets matched via this rule. Action represent the same actions performed on the matched packets in the policy rule either action takes two values in the form of agree to or reject. Packet may be either allowed to enter into the system or either may lead to deny, which are based on some standards [1]. Inaccurate policy is carried out in the firewall when one rule is screen by other rules and the incorrect task of virtual rule ordering. These may create some security inconsistency problem like routing disagreeable traffic and also availability (ease of use) problem like denying genuine traffic which successively affects the firewall performance. Hence this may result in various types of attacks [9] like unauthorized access to the system, denial of service and spoofing like attack to disturb the system result in malicious data to enter into the system.

\section{FIREWALL POLICY ANOMALIES}

There are almost numbers of possible firewall policy anomalies [3] or deviation, which support some of the policy rules that are the following:

Shadowing conflict: - When the packet is inspected with certain condition and action then the rule matches the criteria scheduled which performs the different action. This kind of variation causes the allowed traffic to be ineffective. Therefore, it is important to recognize or either repair the rule which is shadowed and is supposed to occur in firewall policy.

Correlation conflict: - Two are said to be correlated, when the primary packet rule matches the subsequent packet rules and its associate.

Generalization conflict: -When the subsets of packets match up by the rule and also match up previous rule, then there occurs dissimilar actions for the same rule.

Redundancy conflict: - If there is some rules which perform the same action as the other rule perform then there is redundancy conflict. Therefore it results in increase in the Redundancy rule, the space consumptions and time required to investigate. Therefore, it is necessary that redundancy is carried out from the rules and supervisor change the filtering effect so as to reduce the respective level [3].

\section{SYSTEM ARCHITECTURE AND DESIGN}

A framework is designed for detecting the irrelevant anomalies or behavior, so as to enhance security to the system. For defining rules, the administrator first would authenticate. After authentication, the administrator defines the rules which have some condition and action. This is followed up be the next step i.e. anomaly resolution, dynamic re-ordering. At the user end it will select the user and the destination IP port to which the file has to be transferred. And then firewall $\log$ analysis has been done. From that analyze the data and detect $\log$ with irrelevant behavior. The IP which is found to be irrelevant or showing different behavior are blocked with the access. 


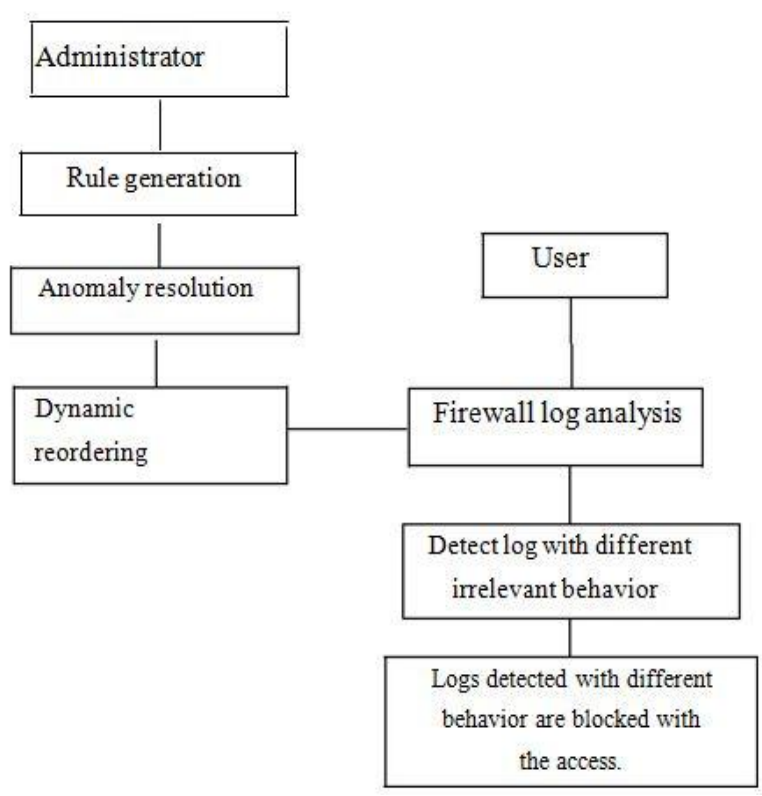

Fig.1. System Architecture

\subsection{Conflict management framework}

The task is divided into either detecting or resolving the difference in firewall policy into framework component; those are explained as the following:

Rule generation: The supervisor defines a rule by giving rule with specific name and a range of fields like s_ address, des address, s_ port, des_ port. One can analyze the entry value depending upon the entry value calculated, certain act can be allowed or denied the numbers of rules that the administrators have entered as a policy in the firewall.

Conflicted rule updating: There are a range of types of firewall policy anomalies which can be exist in the firewall and obstruct the security policy. Any type of inconsistency found in the rule occurs in policy, if so I will be updated. File transformation: The file is chosen which we want to be transferred and then the file is initially process through encryption and later on forwarded to the regulation engine. Rule Engine: Conflict resolution approach obtains mainly the best solution when the entire particular action constraint for each disagreeing segments that is pleased by rearranging the anomaly deviation regulations [3].

\subsection{Policy anomaly resolving and rule-ordering}

The administrator may face difficult problems in solving conflicts which presently occur in the firewall policy anomaly. The configuration process in firewall is important and failure prone. For the firewall policy management a very effective tool is needed so as to manage the conflicts. An efficient approach has been developed on the risk value for conflict detection and resolution strategy [8]. The proposed techniques are adopted to identify the various anomalies occurring in the system. By adopting segmentation technique based on the rules we are able to identify the deviations. We obtained the respective benefits with related to our proposed work:

Conflict Resolution: - The packets which are showing conflicts are discovered at an earlier stage intended for conflict recognition and refinement. These packets are showing conflicts either they are associated with some identified conflicting segments or there may be set of rules and policies that are showing conflict. Since the identified contradictory subdivision, shows associated connection are detected to derive the connected groups for finding the conflict. Then, the problems of conflicts are resolved in which they are found Throughout this correlation process, successively we have to decrease the space which is occupied for searching and either taken for resolving the conflicts exist in the policy.

Action Constraint Generation: - An action constraint defines for each conflicting segments. There are two potential actions that are assigned for a contradictory segment that are either allow or deny and if there exits rules that are showing conflicts. Any packet which goes through the firewall has the specific action that should be taken which describes the contradictory segment by exploit this action constraint. The

Conflicts are identified that are occurring in the policy, for conflicts the risk assessment is performed on firewall policy. The security level is determined based on the vulnerability level within the specified protected network. The risk assessment value is highest, and then the action should be taken either to block or deny the data packets so that data cannot be hampered. Moreover when the risk assessment value is least, then the packet is allowed to pass through the firewall. As this particular constraint method is not affecting in providing the services as given by the network. In addition, the source availability and network services consumption is increased.

Rule Reordering: - The policy rules in the firewall are to pass through a filter. In this proposed technique it deploy

the skew-ness that are identical of firewall rules in order to get better the efficiency of filtering.

Algorithm: Dynamic rule-ordering

1. Input: Set of Rule Ri, Set of Packet Pi

2. Start

3. Initialize $\mathrm{n}:=\mathrm{X}$

4. For every $i=0$ to $R$ do

5. Pi cross- examine with RC ri;

6. If $\mathrm{pi}$ is equivalent $\mathrm{ri}>=\mathrm{x}$ then

7. ri can be logged

8. Else If pi is equivalent $r i<x$ then

9. ri cannot be logged.

10. End if

11. End for

12. End [6]

\subsection{Firewall log analysis}

It would generate a logged data with rare outcomes and repeated rules, which can be used further so as to secure or add more security to the network. Firewall system will generate a large amount of log data and would need policy management framework for dealing with large amount of data [3] [4]. 


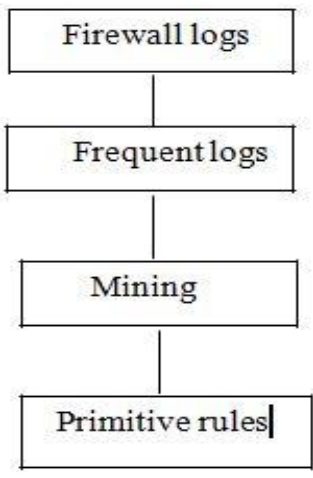

Fig.2. Firewall log analysis

\subsection{Detect Log with Different Irrelevant Behavior}

The Firewall log analysis has been performed which helps us in detecting some irrelevant log data from the gathered data. In this purposed technique, from the log data we can detect the IP which is deviating from its path and showing some irrelevant behavior [2]. For detecting or analyzing the behavior, various types of statistical techniques are used [10].

\subsection{Detected logs are blocked with the access}

To secure the network, the IP which are detected irrelevant are blocked with the access. The data which is captured as unsecure are added in the blocked IP list, as this will enhance more security to the network [10].

\section{IMPLEMENTATION AND RESULTS}

1. Login module

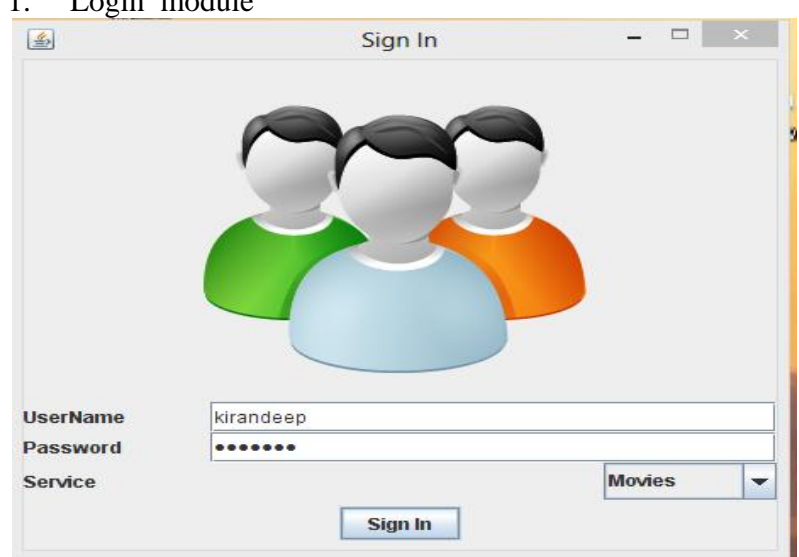

2. User logged in into the system

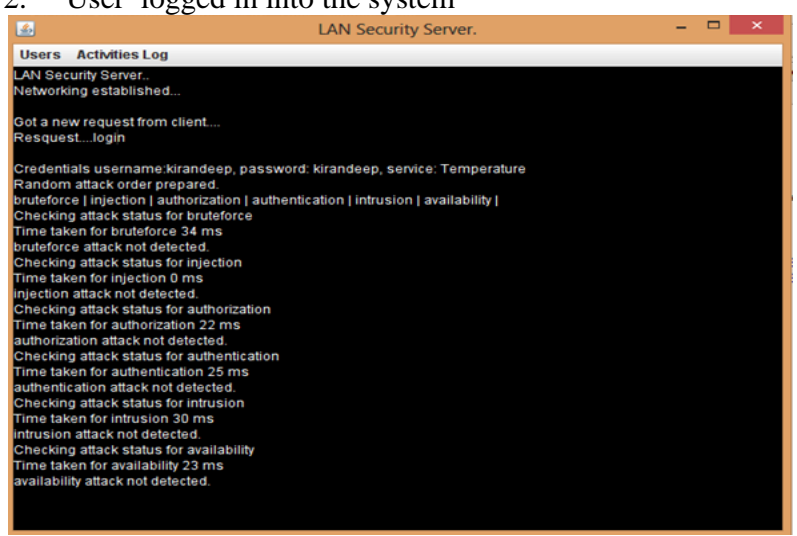

3. Authentication attack

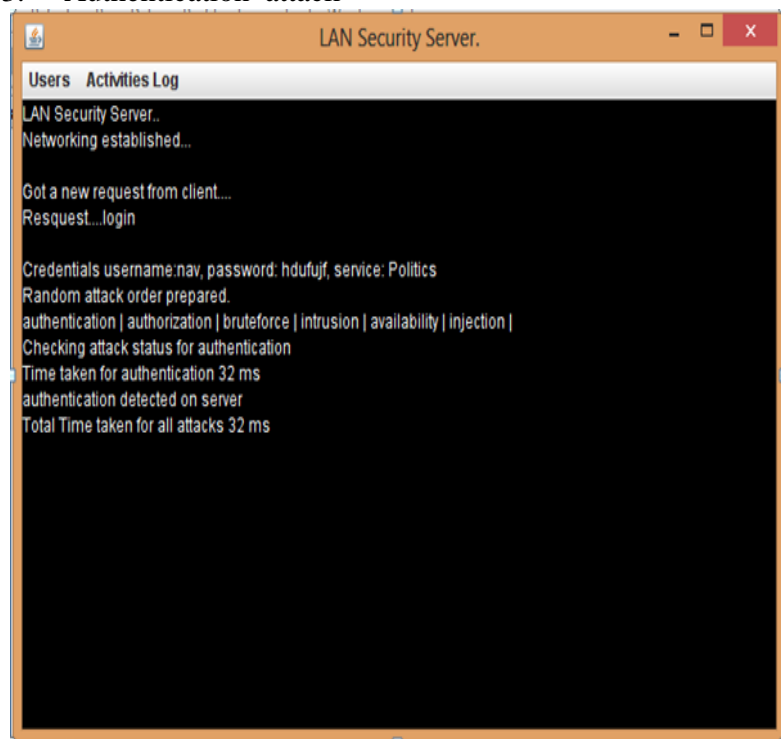

4. Availability attack

\begin{tabular}{l}
\hline LAN Security Server. \\
Users Activities Log \\
LAN Security Server.. \\
Networking established... \\
Got a new request from client.... \\
Resquest...login \\
Credentials username:nav, password: nav, service: News \\
Random attack order prepared. \\
bruteforce | availability | authorization | authentication | intrusion | injection | \\
Checking attack status for bruteforce \\
Time taken for bruteforce $39 \mathrm{~ms}$ \\
bruteforce attack not detected. \\
Checking attack status for availability \\
Time taken for availability $26 \mathrm{~ms}$ \\
availability detected on server \\
Total Time taken for all attacks $32 \mathrm{~ms}$ \\
\end{tabular}

5. Authentication attack

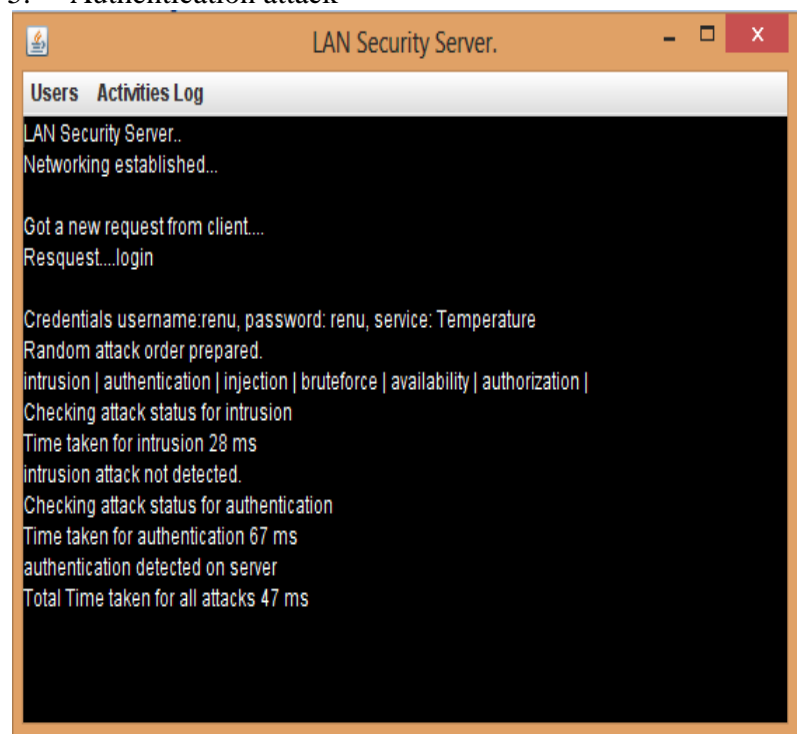


6. Sql injection attack.

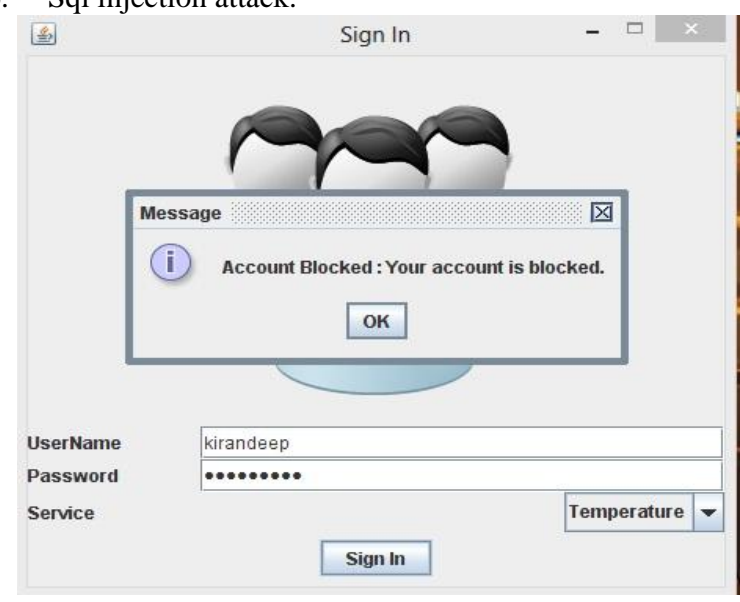

7. User log data gathering

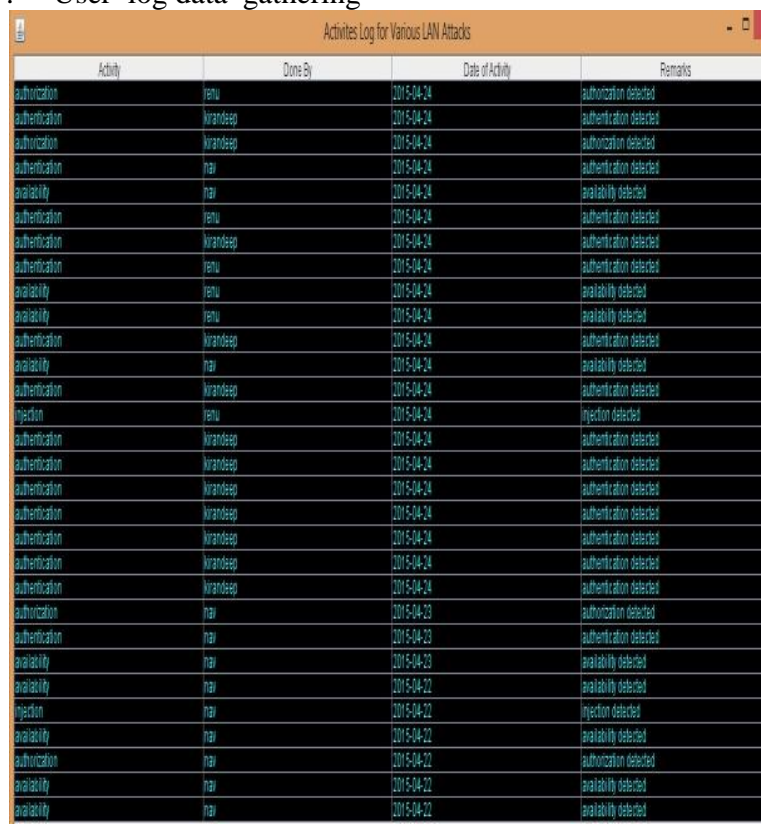

8. Account is blocked

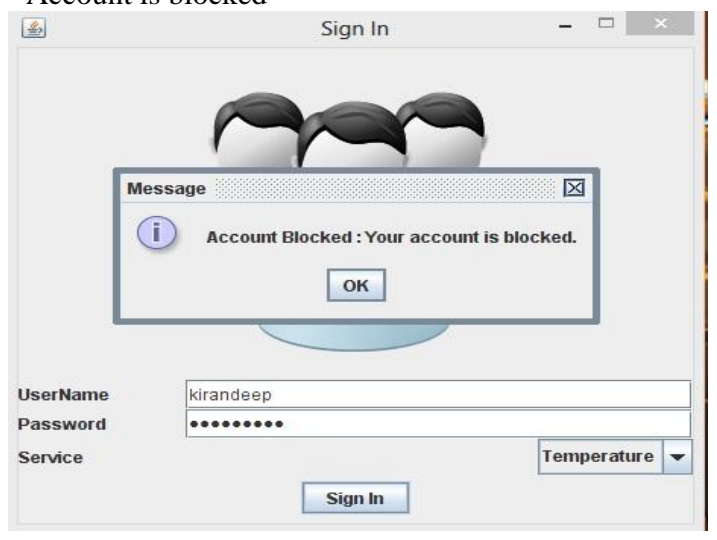

\section{ACKNOWLEDGMENTS}

I would like to take this opportunity to express my deep sense of gratitude to all who helped me directly or indirectly during this work. The encouragement and critics are sources of innovative ideas, inspiration and cause behind the successful completion of this research paper. I am highly obliged to all faculty members of computer science and engineering department for their support and encouragement. I would like to express my sincere appreciation and gratitude towards my friends for their encouragement, consistent support and invaluable suggestions at the time I needed the most.

\section{REFERENCES}

[1].ASARCIKLI,s.(october 2005). firewall monitoring using intrusion detection system.

[2].Design and implementation of content firewall. (n.d.). university of wollongong , 13

[3].Gawanmeh, A. (2014). automatic verification of security policies in firewalls with dynamic rule sequence. internation conference on information systems , 6 .

[4].Inc, d. s. (2012). Introduction to Firewall . intelligent edu.com , p. 4.

[5].kakuru, S. (2011). behavior based network traffic analysis tool. IEEE, 4.

[6].Kavitha karun A, 1. k. (2013). Firewall log analysis and dynamic rule re-ordering in firewall policy anomaly management. IEEE , 4.

[7].Kazimierz Kowalski, M. B. (2006). Analysis of Log files Intersections for security Enhancement. IEEE, 5.

[8].kumar, s. (2012-14). Firewall. techno sticker .

[9].Lubana k, R. c. (2013). A study on firewall policy anomaly representation techniques. ijarcce, 4 .

[10].Mukupa, I. M. (November 8, 2010). firewall rule set optimization. In firewall rule set optimization (p. 90). Grahamstown, South Africa.

[11].R.sherman. (2000). computer security.

[12].Rupali chaure, s. k. (2010). firewall anomaly detection and removal techniques. IEEE, 4.

[13].Sandip k pal, m. a. (2014). User based behavior anomaly detection. integrity learning responsibility.

[14].search, d. (2012). different types of attacks. intelligent edu.com , p. 3 .

[15].Schuba, C. L. (December 1997). modelling,design and implementation of firewall technology. Purdue University.

[16].Teddy Mantoro, N. A. (2013). Log Visualization of Intrusion and Prevention Reverse Proxy Server Against Web attacks. ieee , 5 . 